\title{
Experimental Study on the Measurement of Water Bottom Vibration Induced by Underwater Drilling Blasting
}

\author{
Gu Wenbin, ${ }^{1}$ Chen Jianghai, ${ }^{1,2}$ Wang Zhenxiong, ${ }^{1}$ Wang Zhihua, \\ Liu Jianqing, ${ }^{1}$ and Lu Ming ${ }^{4}$ \\ ${ }^{1}$ College of Field Engineering, PLA University of Science and Technology, Nanjing 210007, China \\ ${ }^{2}$ Unit No. 65307 of PLA, Jilin 132002, China \\ ${ }^{3}$ School of Electronic and Optical Engineering, Nanjing University of Science and Technology, Nanjing 210007, China \\ ${ }^{4}$ Wuhan Ordnance N.C.O. School, Wuhan 430075, China
}

Correspondence should be addressed to Chen Jianghai; chenjianghai@yahoo.com

Received 4 March 2015; Accepted 19 August 2015

Academic Editor: Mickaël Lallart

Copyright (c) $2015 \mathrm{Gu}$ Wenbin et al. This is an open access article distributed under the Creative Commons Attribution License, which permits unrestricted use, distribution, and reproduction in any medium, provided the original work is properly cited.

\begin{abstract}
Due to the lack of proper instrumentations and the difficulties in underwater measurements, the studies about water bottom vibration induced by underwater drilling blasting are seldom reported. In order to investigate the propagation and attenuation laws of blasting induced water bottom vibration, a water bottom vibration monitor was developed with consideration of the difficulties in underwater measurements. By means of this equipment, the actual water bottom vibration induced by underwater drilling blasting was measured in a field experiment. It shows that the water bottom vibration monitor could collect vibration signals quite effectively in underwater environments. The followed signal analysis shows that the characteristics of water bottom vibration and land ground vibration induced by the same underwater drilling blasting are quite different due to the different geological environments. The amplitude and frequency band of water bottom vibration both exceed those of land ground vibration. Water bottom vibration is mainly in low-frequency band that induced by blasting impact directly acts on rock. Besides the low-frequency component, land vibration contains another higher frequency band component that induced by followed water hammer wave acts on bank slope.
\end{abstract}

\section{Introduction}

Like land blasting, underwater drilling blasting, which is being used in onshore or offshore constructions more and more widely, also has many considerable impacts on surrounding environment. Blasting vibration is one of the hazards induced by underwater blasting project. The characteristics of underwater blasting and the propagation laws of induced vibration are both different from those of land blasting due to the influence of underwater environments such as water mass, water-saturated sediments, and rock mass $[1,2]$. However, current study of underwater blasting vibration mainly concentrates on the response of land ground and structures [3-5]. Researches about induced water bottom vibration are very few, and existing achievements mostly focused on theoretical analysis or are predicted from land ground tests which need to be verified by actual water bottom measurements. That is because of the lack of proper water bottom instrumentations and the much more difficulties in measurements in underwater than on land ground or structures. But with the progress of economics and technology development, more and more large underwater lifeline projects have been constructed, such as submarine tunnels, subsea communication cables, and oil-gas pipelines [6-8], and play very important role in our society. These projects are so essential that they cannot be injured by any hazards, including the water bottom vibrations induced by natural earthquake and underwater blasting. For those projects around the underwater blasting area, to monitor the blasting induced underwater vibrations is particularly necessary. Therefore, it is very important and valuable to develop proper instrumentations and carry out filed measurements to master the propagation and attenuation laws of induced water bottom vibrations for the improvement of practical underwater blasting project and the safety of large lifeline projects. 
Presently, there are some equipment and methods to measure ocean bottom seismic waves in natural earthquake research field. Ocean Bottom Seismometer (OBS) $[9,10]$ can work several months in $6 \mathrm{~km}$ depth ocean bottom to measure seismic waves ranging from $0 \mathrm{~Hz}$ to $30 \mathrm{~Hz}$. But OBS is directly placed on the seafloor and is always influenced by seabed topography, sediment layer, and ocean turbulence [11, 12]. The Neath Seafloor Equipment for Recording Earth's Internal Deformation (NEREID) used in the Ocean Discovery Program (ODP) [13, 14] is the most advanced water bottom seismological observatory system, which installs seismometer in seafloor borehole constructed by the Riser Drilling Vessel to improve the quality of seismic observation. It works in ocean bottom rock $2.5 \mathrm{~km}$ in depth for several years and observes seismic motion below $50 \mathrm{~Hz}$. However, both OBS and NEREID are expensive owing to their complicated components and complex installation process, which are mainly applied in long-term and large-scale deep ocean geological investigation to detect natural seismic waves in low or even low-frequency band. And most observing stations are hundreds or thousands of kilometers away from epicenter. Obviously, they are not suitable but can provide good reference to the measurements of broadband (up to $500 \mathrm{~Hz}$ ) and near (tens or hundreds of meters away from epicenter) water bottom vibrations induced by short-term (days) and shallow (deep to 100 meters) underwater drilling blasting.

In this paper, a water bottom vibrometer (WBV) and relevant testing process were developed in consideration of the difficulties in underwater measurements. By using this technique, the actual water bottom vibrations of underwater blasting were measured and compared with that of land ground vibration that is at the same horizontal distance from the same blasting center.

\section{Development of WBV}

2.1. Performance Requirement. Water bottom vibration measurement is to measure vibration by using a transducer attached to water bottom, which is much more difficult compared with the land blasting vibration measurement.

The first difficulty is the strict waterproof requirement on sensors and electric cables. As wireless electromagnetic signal can hardly be transmitted in water and acoustic signal is liable to be concealed by blasting noise, present underwater measurement usually uses electric cables to transmit signal from underwater sensor to land data collector [12]. The underwater sensors and electric cables must be strictly waterproofed; otherwise the measurement might fail once the instrumentations are out of work because of circuit short.

Secondly, the way of installing seismometer to water bottom is very hard. Usually, underwater blasting measurements sensors are installed by frogman to underwater gauging point. But the water bottom environment is very complex and the bottom water would be much more turbid during underwater drilling operation. So the traditional installation method by manual work is very dangerous and costly.

Thirdly, the fastness between seismometer and water floor is difficult. To get accurate water bottom vibration signal, the fastness between seismometer and water floor must be

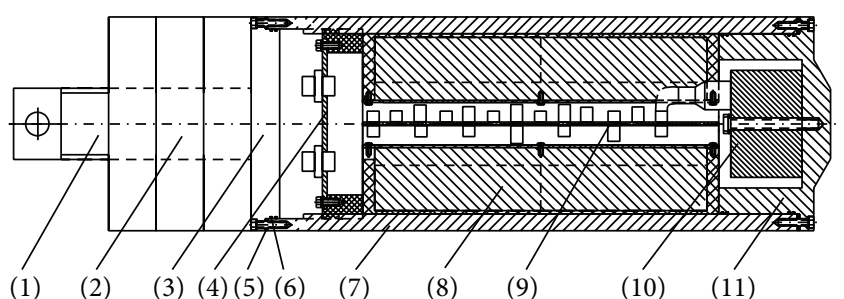

(1) (2)

(3) (4) (5) (6) (7)

(8)

(9)

(10)

(11)

(1) Guide rod

(2) Positioning block

(3) Head cover

(4) Interface module

(5) Seal gasket

(7) Housing

(8) Power module

(9) Central control module

(6) Seal ring

(10) Accelerometer

(11) Base

FIGURE 1: Structure diagram of water bottom vibrometer.

firm. But the natural water floor is usually covered with break stones or silt sediments and the sensors are used to be placed on water floor or plugged in sediments. The signals frequency band detected from sensors would be incomplete due to the low-frequency passing characteristic of sediments. The best situation is fixing seismometer on water bottom base rock to assure the continuous displacement of seismometer and base rock, and the seismometer should be taken back easily after measurement for reusing next time.

The last problem is the serious noise disturbance in signal. Without firm fastness between seismometer and water floor, and with the use of electric cables, water turbulence and shock waves induced by underwater blasting would exert bad influences on sensors and cables and bring serious noise in collected signals.

In a word, besides the general performance of land seismometer, water bottom vibration measurement instrumentations should have some special abilities suited for underwater circumstances, such as well enclosing, water proofing, noise resistance, stable operation, convenient installation, and firm fastness on water bottom.

2.2. Structure and Circuit Principle. Learning from the performance of existing common vibrometer for land blasting and considering the requirements of underwater blasting vibration measurements, an autonomous WBV was developed, as shown in Figure 1. It mainly consists of guide rod, positioning block, head cover, interface module, seals, housing, power module, central control module, transducer, and base. After all modules assembled, the inside space would be filled with epoxy resin.

The shell surface was hardened to resist corrosion and oxidation. The cover and the housing are connected by screws and sealed with rubber gaskets and rings between the joint surfaces to guarantee fully waterproof effect. Positioning block is used to locate housing in underwater borehole to ensure the stance of vibrometer and there are several specifications that could be selected according to the corresponding size of the drill diameter. Guide rod and positioning block are connected by screw thread. There is an aperture on guide rod tip to connect cables and floats. Interface module, 


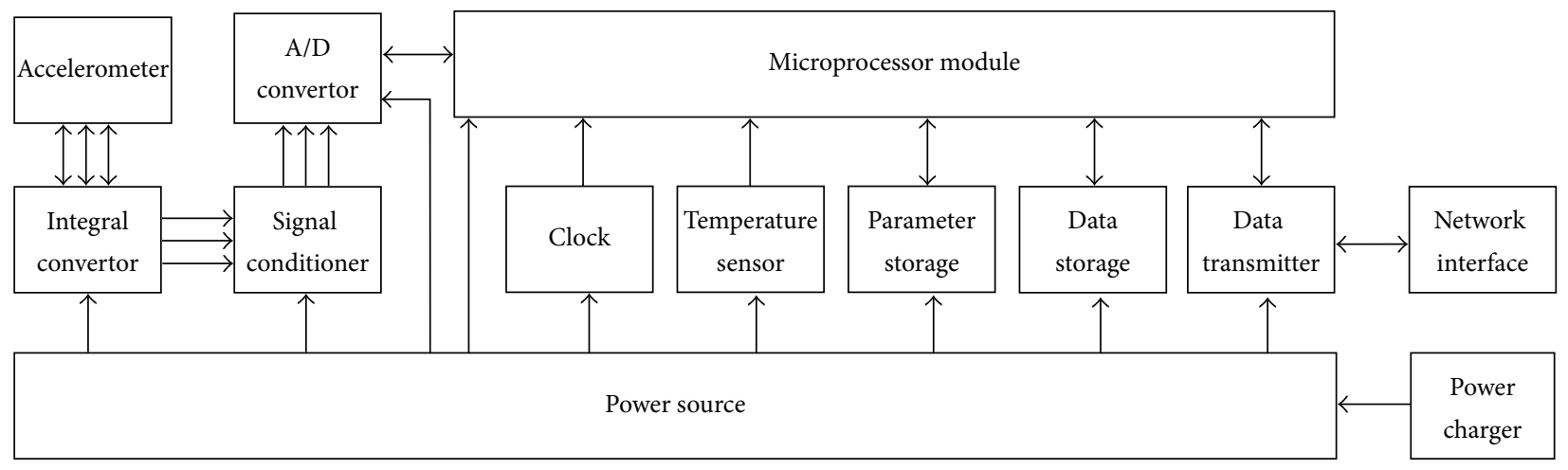

FIgURE 2: Circuit function schematic.

consisting of connector panel, power switch, power charging port, network interface, and status indicator lights, is used to interact between internal system and external manipulation. Power source module, composed of rechargeable lithium cells connected in parallel, supplies power for the entire system up to 24 hours. In order to adapt to the minimum drill size used in general underwater blasting, the interior and exterior spaces occupied by instrument parts must be minimized. But general magnetic speed sensor volume was relatively large, so a three-orientation ICP piezoelectric accelerometer with relatively smaller size is used as transducer module and is rigidly fixed in the base with a stainless steel screw to receive rock vibrations.

Central control module consists of several submodules, such as integral converter, signal conditioner, analog-todigital (A/D) converter, microprocessor, parameter storage, data storage, data transmission, clock, and temperature sensor. Integral converter submodule is composed of TL062 amplifier chips and band-pass filter circuit which is used to convert acceleration signal from accelerometer to speed signal. Signal conditioner submodule is an emitter follower circuit, composed of OPA417 operational amplifiers, and is used to improve input impedance and increase drive capacity. Analog-to-digital converter submodule is composed of AD7656 chips and peripheral circuits; it is used to digitalize signal before storage to memory chips. Microprocessor submodule is composed of ARM processor, clock circuit, and JTAG circuit and is used to control and coordinate entire acquisition system. Parameter storage submodule stores system setup parameters, including the necessary basic parameters and status parameters for system operation. All the data after analog-to-digital conversion are stored to the data storage submodule with $2.56 \mathrm{M}$ bytes FRAM memory. Data transmission submodule, composed of network protocol chip, isolation transformers, and so forth, transmits data between acquisition systems and host computer using Ethernet transmission technology. Clock module for realtime clock can be set by software to realize such function as sleeping, waking up, and starting acquisition. Temperature sensor submodule is used to monitor internal temperature, so the power would be cut off automatically once the temperature exceeds the predetermined limit to protect internal circuit and data security.
Circuit function schematic of the system is shown in Figure 2. Three-orientation vibration acceleration information is converted to a three-channel voltage signal in dynamic range $-5 \mathrm{~V} \sim+5 \mathrm{~V}$. After integral conversion and signal conditioning, the three-channel acceleration signal is transmitted to velocity signal in ranges $-10 \mathrm{~V} \sim+10 \mathrm{~V}$. By analog-to-digital conversion and acquisition process, the analog voltage of velocity signal will be transmitted to the corresponding digital signal and stored in data storage module. By data transmission submodule, stored data can be sent via the network interface to host computer for subsequent analysis and processing. The host computer also set the system's parameters via network interface and data transmission submodule. The power source module converts the $3.7 \mathrm{~V}$ output from lithium battery pack to 6 kinds of voltage powers for each subcircuit, such as providing analog $\pm 12 \mathrm{~V}$ for accelerator and conditioning circuit, providing digital $+3.3 \mathrm{~V}$, digital $+5 \mathrm{~V}$, analog $+5 \mathrm{~V}$ for analog-to-digital conversion circuit, providing digital $+3.3 \mathrm{~V}$ for microprocessor module and memory module, and providing digital $+3.3 \mathrm{~V},+1.8 \mathrm{~V}$ for data transmission module.

2.3. Main Features. Considering the complex environments in underwater vibration test, the WBV has the following advantages.

The first one is corrosion-resistant and waterproof performance. By adopting multichannel sealing measures, the shell is well waterproofed. Field water tightness test shows that the housing can work well in continuous 24 hours without leakage in the water of minimum 50-meter depth. After surface hardening and antioxidant processing, the aluminum shell is also corrosion-resistant and impact-resistant.

The second one is the convenience of setting and using. Due to the integrated designation of accelerometer, power, control, and acquisition circuits, the WBV has more simplified structure compared with general separated vibrometer and is very convenient to install and fetch back.

The third one is the high reliability of electric circuit. Because of the automatic sleeping and waking up timer function, the WBV can start automatically according to the preset time, trigger automatically according to the preset threshold, and work stably with independent acquisition and multisection storage. With high-quality circuit layout technique, it 
TABLE 1: Basic performance indicators.

\begin{tabular}{|c|c|c|c|c|c|}
\hline Input channel & $X, Y, Z$ & Sensitivity & $28 \pm 10 \% \mathrm{~V} / \mathrm{m}^{-1} \mathrm{~s}^{-1}$ & $\mathrm{~A} / \mathrm{D}$ resolution & 16 bits \\
\hline Frequency range & $5-500 \mathrm{~Hz}( \pm 3 \mathrm{db})$ & Scale range & $0.005-0.35 \mathrm{~m} / \mathrm{s}$ & Sampling frequency & $2 \mathrm{k}-8 \mathrm{kHz}$ \\
\hline Trigger pattern & On threshold & Trigger level & $0.05 \mathrm{~V}-2 \mathrm{~V}$ & Signal sections & 10 \\
\hline Power supply & $\begin{array}{c}\text { Rechargeable lithium } \\
\text { batteries }\end{array}$ & Battery life & 24 hours & Time per section & $5 s-20 s$ \\
\hline Overall dimensions & $\Phi 93 \mathrm{~mm} \times 340 \mathrm{~mm}$ & Working temperature & $-20^{\circ} \mathrm{C}-80^{\circ} \mathrm{C}$ & Negative delay time & $-100 \mathrm{~ms}$ \\
\hline Shell material & $\begin{array}{l}\text { Duralumin (surface } \\
\text { hardening) }\end{array}$ & Total weight & $\begin{array}{c}3 \mathrm{~kg} \text { (excluding positioning } \\
\text { block) }\end{array}$ & Water depth & $\leqslant 50 \mathrm{~m}$ \\
\hline
\end{tabular}

also has stable performance of high speed data transmission in running state and reliable data holding in power-off state.

The fourth one is the high quality of data collection. Without the use of underwater cables to transmit signals, the noises caused by blasting shock on cables can be greatly eliminated; the vibrometer, placed in rock borehole, directly feels the vibration in bedrock and would not be affected by bottom sediments and water flow; at the same time, the vibration signal is locally digitized and immediately collected and stored, so the signal-to-noise ratio is relatively higher.

At last, the performance meets the requirements of present blasting vibration testing. With reference to the general land seismometers and with redundant designation, the performance of WBV can meet the basic requirements of underwater blasting vibration tests.

After calibration on lab vibrator and field testing in underwater, the basic performance indicators are acquired and shown in Table 1.

\section{Water Bottom Vibration Test}

Combined with the vibration monitoring project of underwater drilling blasting in Shanghai Yangshan Port, a water bottom vibration testing trial was carried out. For comparison, a TC-4850-type land vibrometer developed by the Chengdu Branch of Chinese Academy of Sciences was used to measure land ground vibration simultaneously.

3.1. Test Parameters and Geological Environment. Underwater blasting region was $40 \mathrm{~m}$ distant from bank and had 23 blasting boreholes totally in two rows; rows interval was $2.5 \mathrm{~m}$, and boreholes step was $2 \mathrm{~m}$; total charge was $765 \mathrm{~kg}$. The maximum segment charge was $76.5 \mathrm{~kg}$, bedrock borehole depth was $7.8 \mathrm{~m}$, bore diameter was $0.165 \mathrm{~m}$, charge length was $6.8 \mathrm{~m}$, and packing length was $1 \mathrm{~m}$.

According to the circumstance of local site, the TC-4850 land vibrometer was placed at land ground point north to the underwater blasting area, and the WBV was placed in a water bottom borehole west to the underwater blasting area. The sampling frequency of TC-4850 was $8 \mathrm{kHz}$ and the trigger threshold was $0.03 \mathrm{~cm} / \mathrm{s}$. The sampling frequency of WBV was $8 \mathrm{kHz}$ and the trigger threshold was $0.05 \mathrm{~V}(0.175 \mathrm{~cm} / \mathrm{s})$.

Both land and underwater measuring points were $50 \mathrm{~m}$ horizontally distant away from the center of blasting area. The vertically average altitude from land measuring point to underwater blasting district was $15 \mathrm{~m}$. The land point geology roughly included three layers: from top to bottom were gravel layer, macadam layer, and rock mass. The water depth of underwater measuring point was $10 \mathrm{~m}$; the installation hole on bedrock was $0.5 \mathrm{~m}$ in depth and $0.165 \mathrm{~m}$ in diameter. The water bottom geology also roughly included three layers: from top to bottom were $3 \mathrm{~m}$ thick silt layer, scattered macadam layer, and rock mass, as shown in Figure 3.

3.2. Testing Process. The process of water bottom vibration testing includes the following steps.

First step is underwater drilling. Drill a $0.5 \mathrm{~m}$ depth shallow hole on bedrock outside the blasting area by using the submersible drilling rig on the underwater drilling ship. After clearing debris in drill hole with high-pressure air, extract the drill bit without moving the casing pipe.

Second step is the WBV parameter setting. In the meantime of drilling, connect the data interface on WBV via a dedicated data line to PC and set parameters at the interface software, such as the initial start-up time, the trigger threshold, the sampling frequency, the silent time, and acquisition times.

Third step is the WBV assembly and float connection. After parameter setting, close the head cover with screws and reliable seal, install proper positioning block according to corresponding drill size to the guide rod, and connect a float via a rope to the top hole of guide rod to mark location in water. Connect an orientation adjusting pole on the rings and make the direction line of the adjusting pole accord with the $x$-axis of WBV.

Fourth step is the WBV fixing. Place the WBV in the bedrock hole through the casing pipe; rotate the adjusting pole to make sure the direction line and $x$-axis of WBV both orient to the blasting center. Withdraw the adjusting pole and casing pipe and remove the drilling ship. The WBV was limited circumferentially and vertically within the drilling hole by the positioning block and surrounding sediments that backfilled to the hole. So the WBV was firmly fixed in the hole to ensure close contact between the base of WBV and the bottom of drilling hole, as shown in Figure 4.

Fifth step is water bottom vibration signal acquisition. The WBV start up automatically at preset initial time before underwater blasting. When water bottom vibration amplitude reaches the preset threshold, the WBV was triggered and starts collecting the signals of underwater rock vibration. After acquisition, the WBV turns into low power dissipation state of silence and waiting for next blasting event.

The last step is fetching the WBV back and reading data. After underwater blasting, fetch the WBV back by float 


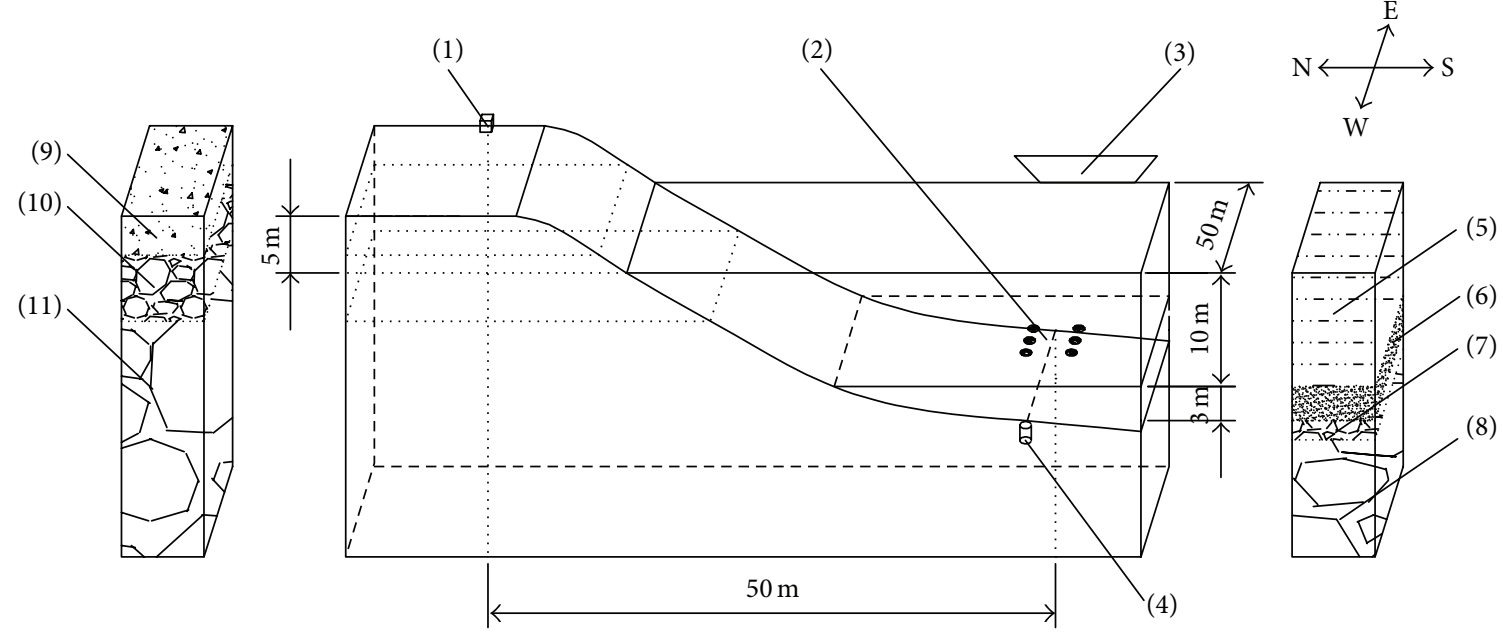
(7) Macadam
(8) Rock mass
(9) Gravel
(10) Macadam
(11) Rock mass

FIgURE 3: Measuring circumstance diagram.

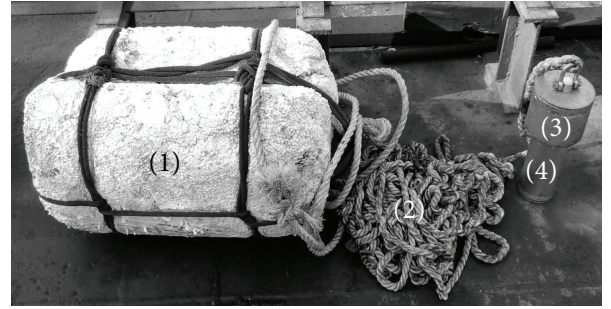

(a)

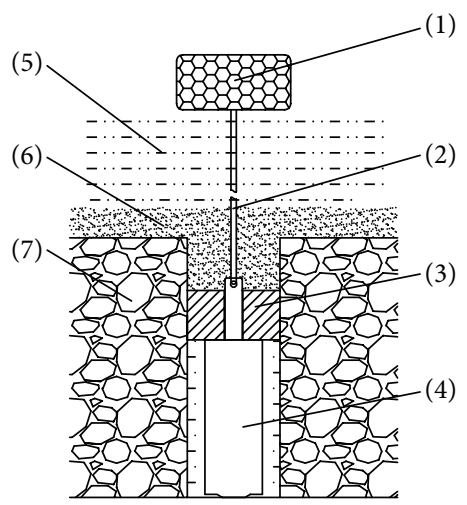

(b)

Figure 4: WBV setting diagram. (1) Float, (2) rope, (3) positioning block, (4) WBV, (5) water, (6) silt, and (7) base rock.

and rope, remove the surface mud and water stains, open head cover, connect the data interface of WBV via dedicated data cable to PC, and read data by interface software for subsequent analysis.

3.3. Testing Results. We observe after fetching the WBV back that the instrument housing is intact without internal leakage and each module operates very well.

By using the MATLAB software, the acquired signal was analyzed in time domain and frequency domain. The peak velocity and main frequency of water bottom point vibration induced by underwater drilling blasting are shown in Table 2; the signal waveform and FFT spectrum are shown in Figure 5.

The peak velocity and main frequency of land point vibration are shown in Table 3; the signal waveform and FFT spectrum are shown in Figure 6.
TABLE 2: Peak velocity and main frequency (WBV).

\begin{tabular}{lccc}
\hline Number & Channel & Peak velocity & Main frequency \\
\hline 1 & $X$ & $9.664 \mathrm{~cm} / \mathrm{s}$ & $36 \mathrm{~Hz}$ \\
2 & $Y$ & $5.904 \mathrm{~cm} / \mathrm{s}$ & $47 \mathrm{~Hz}$ \\
3 & $Z$ & $20.212 \mathrm{~cm} / \mathrm{s}$ & $36 \mathrm{~Hz}$ \\
\hline
\end{tabular}

TABLE 3: Peak velocity and main frequency (TC-4850).

\begin{tabular}{lccc}
\hline Number & Channel & Peak velocity & Main frequency \\
\hline 1 & $X$ & $1.251 \mathrm{~cm} / \mathrm{s}$ & $26 \mathrm{~Hz}$ \\
2 & $Y$ & $2.199 \mathrm{~cm} / \mathrm{s}$ & $26 \mathrm{~Hz}$ \\
3 & $Z$ & $2.765 \mathrm{~cm} / \mathrm{s}$ & $26 \mathrm{~Hz}$ \\
\hline
\end{tabular}



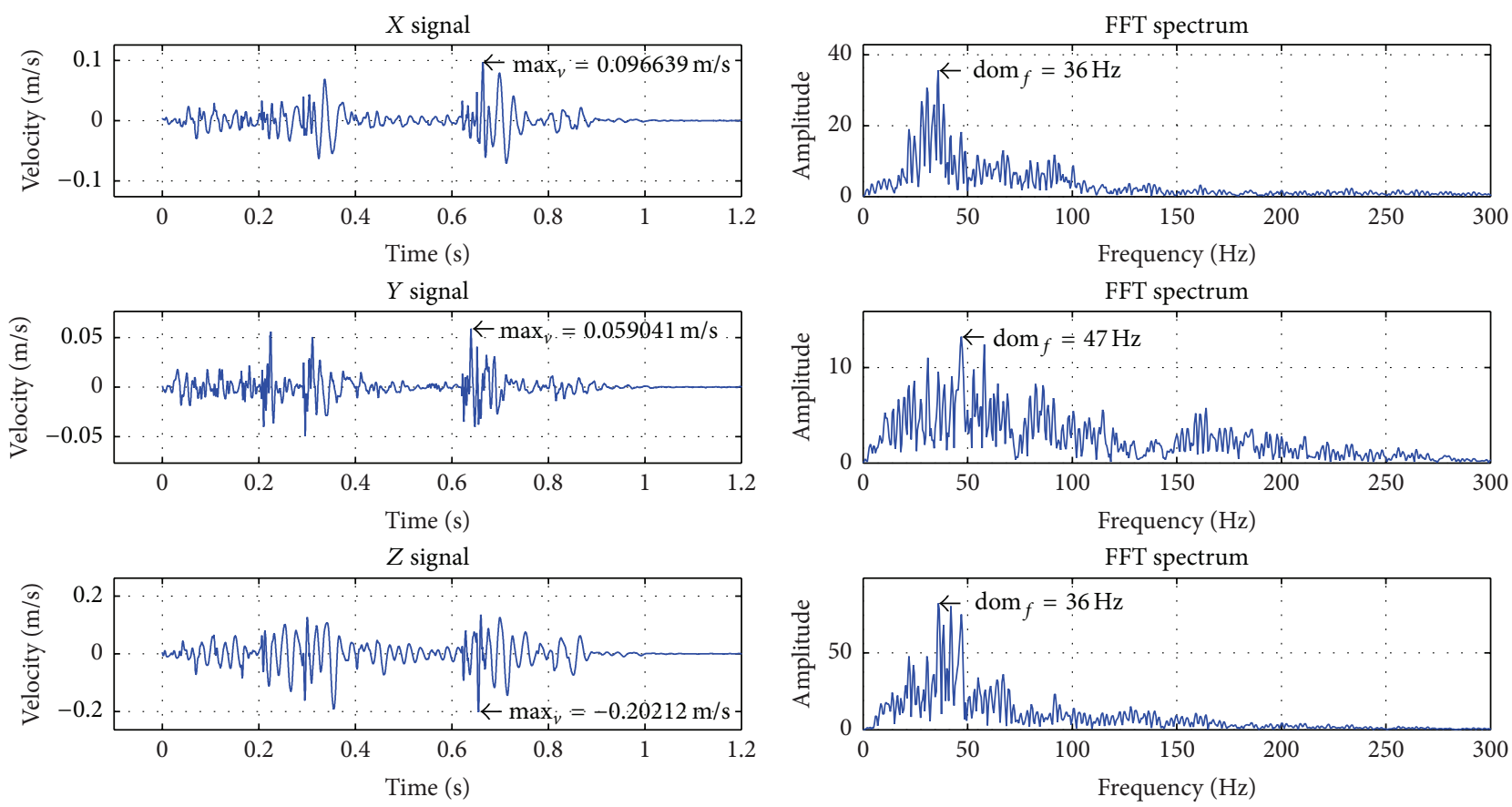

FIGURE 5: Waveform and spectrum (WBV).
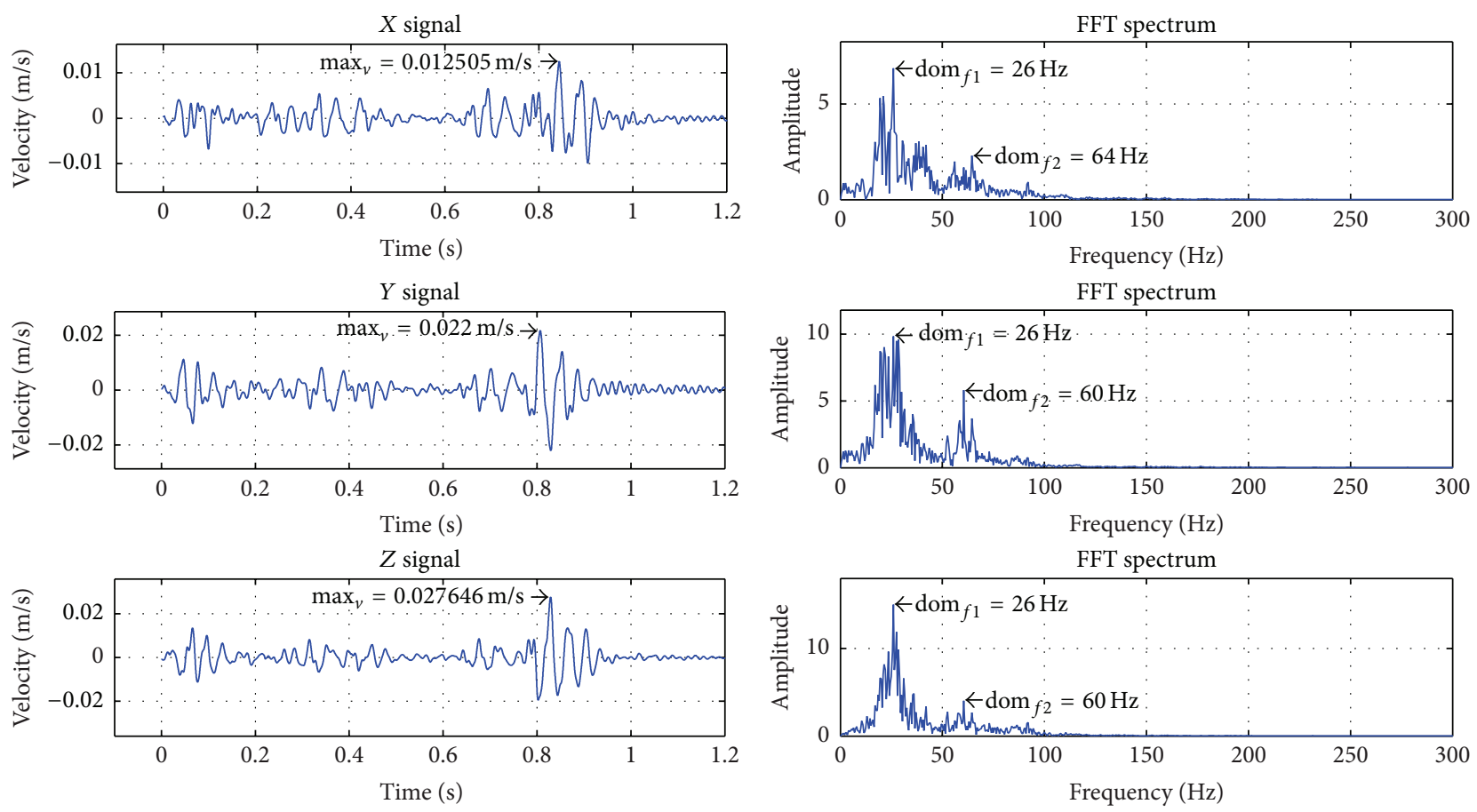

Figure 6: Waveform and spectrum (TC-4850).

\section{Data Analysis}

It is shown that the waveforms acquired from TC-4850 and WBV are very similar, but the peak velocity values and duration time are distinctly different. The vertical vibration peak amplitude attenuates $86.32 \%$ from $20.212 \mathrm{~cm} / \mathrm{s}$ at water bottom point to $2.765 \mathrm{~cm} / \mathrm{s}$ at land point. The horizontal vibration peak amplitude attenuates $87.05 \%$ at $x$-axis and $62.75 \%$ at $y$-axis separately. The duration time of water bottom vibration is $0.9 \mathrm{~s}$ but that of the land vibration is almost $0.95 \mathrm{~s}$. The time of peak amplitude delays $0.2 \mathrm{~s}$ from water bottom point to land point. The land vibration 
clocked is $26 \mathrm{~Hz}$ and frequency ranges below $100 \mathrm{~Hz}$; the water bottom vibration clocked is within $36 \mathrm{~Hz} \sim 47 \mathrm{~Hz}$ and frequency ranges below $300 \mathrm{~Hz}$ and has a subfrequency band around $60 \mathrm{~Hz}$. In a word, the peak amplitude of water bottom vibration is larger, the main frequency is higher, and the frequency band is wider than that of land vibration.

The signal characteristics can be explained by the generation and propagation mechanism of underwater blasting vibration.

As to the generation mechanism of underwater blasting vibration, since the underwater explosive is covered and surrounded by water and blocked by thick sedimentary layers, the bind effect of bursting is greatly enhanced and the seismic intensity of underwater blasting is much larger than that of land blasting. Relative research has proved that [3] the land seismic waves generated by underwater blasting mainly consisted of two parts: the first was the low-frequency seismic waves generated by explosions direct role on the rock, followed by the high-frequency seismic waves generated by water oscillation on the bottom and slope induced by underwater explosion shock wave.

For the relative positions of water bottom point and land ground point, the horizontal distances between every measuring point and blasting center are both 50 meters, but there is 15 -meter height difference, just on the bottom and top of a slope with high-wide ratio $3 / 10$. So the straight distance between land measuring point and underwater blasting center increased to 52.2 meters. The distance of surface wave spread from blasting center along the slope to land measuring point is greater than distance from blasting center along the bottom plane to underwater measuring point, while the vertical vibration propagation distance from blasting center to land measuring point increased 15 meters compared to underwater measuring point. The study has shown that [15] there was a slope effect when seismic waves propagated along slope from bottom to top point: when the gradient was greater than $1 / 2$, the vibration at top point was greater than that of bottom point and the elevation amplification effect was very obvious; when the gradient was less than $1 / 2$, the effect of elevation amplification would be smaller than that of distance attenuation. As to the above field testing, the slope gradient is less than $1 / 2$, so the elevation amplification does not exist and the vibration attenuation with the increases of distance is very obvious.

Again, in the case of vibration propagation medium, the influence of geological conditions on seismic wave propagation is very great $[16,17]$. The geological differences under two measuring points are very obvious (Figure 3 ). For water floor circumstance, because the upper viscous sedimentary layer and covered water are very thick, blasting vibration energy is mainly spread through the rock medium. And the underlying rock is homogeny and is water-saturated, so the elastic wave propagates speedily and attenuates slowly. The vibration amplitude measured in bedrock borehole is very large; the frequency band is wide. The vibration component is mainly induced by the explosion that acts directly on rock. For land circumstance, besides the bottom rock mass layer, the middle macadam layer and upper gravel layer are both thick and dry with serious fracture and heterogeneity. So the medium absorption and attenuation effects on blasting seismic wave especially to high-frequency components are very significant. Meanwhile, the scope and impact strength of deep water oscillation on slope are very large and the frequency is higher. The synthetical results of these effects are that the vibration amplitude of land measuring point is much smaller than that of water bottom point and contains $26 \mathrm{~Hz}$ low-frequency component that induced by explosion acts directly on rock and $60 \mathrm{~Hz}$ high-frequency component that is induced by water hammer wave on the slope.

\section{Conclusions}

In this paper, an independent acquisition WBV that could adapt to complex underwater environments was developed in consideration of the difficulties in underwater measurements and with reference to the performance of general land blasting seismometers. Combined with the vibration monitoring project of underwater drilling blasting, a field test was carried out and the actual water bottom vibrations were measured and analyzed. Some conclusions can be presented as follows:

(1) With reference to general land vibrometer and design based on the consideration of difficulties in underwater testing, the WBV can operate stably and get high-quality water bottom vibration signal during underwater blasting.

(2) Data analysis shows that, because of the geological differences of water bottom and land ground, the characteristics of vibration signals collected at two points are different obviously. The water bottom vibration in three directions has larger amplitude, higher main frequency, and wider frequency band than the land ground vibration induced by the same underwater blasting and at the same horizontal distance from epicenter. Water bottom vibration is mainly in low frequency that is induced by blasting effects which directly acts on rock. Land vibration contains two components, the $26 \mathrm{~Hz}$ low-frequency component induced by explosion effect that acts directly on rock and the $60 \mathrm{~Hz}$ high-frequency component induced by water hammer wave that acts on slope.

\section{Conflict of Interests}

The authors declare that there is no conflict of interests regarding the publication of this paper.

\section{Acknowledgments}

The study is partly supported by NSFC (no. 51175511). The underwater blasting site was provided by the Blasting Services Division of Nanjing Institute of Engineering Corps and supported by chief engineer Yang Zhixu. This paper got careful proofreading from Dr. He Long. The authors are grateful to those persons and organizations for their invaluable help. 


\section{References}

[1] R. Cole, Underwater Explosions, Dover Publications, New York, NY, USA, 1965.

[2] A. V. Adushkin, V. N. Burchik, A. I. Goncharov, V. I. Kulikov, B. D. Khristoforov, and V. I. Tsykanovskii, "Seismic, hydroacoustic, and acoustic action of underwater explosions," Combustion, Explosion and Shock Waves, vol. 40, no. 6, pp. 707-713, 2004.

[3] J.-Y. Tong, J.-W. Shi, C.-H. Xiong, and Z.-Y. Zhang, "Study on the law of influence of underwater engineering blasting on environment (II)," Blasting, vol. 17, no. 4, pp. 1-4, 2000 (Chinese).

[4] C. K. Madheswaran, R. Sundaravadivelu, A. Boominathan, and K. Natarajan, "Response of berthing structure due to underwater blasting for rock dredging," Experimental Techniques, vol. 33, no. 3, pp. 61-72, 2009.

[5] D.-W. Zhong, "Seismic test and analysis of underwater drilling blasting for Taiziji waterway," Journal of Wuhan University of Science and Technology, vol. 34, no. 5, pp. 350-353, 2011 (Chinese).

[6] H.-P. Wang, S.-C. Li, and L. Qiao, "Dynamic research on the minimum rock covers of subsea tunnel construction using drilling and blasting method," Rock and Soil Mechanics, vol. 27, pp. 290-295, 2006 (Chinese).

[7] A.-W. Liu, "Earthquake effects on submarine cables," Recent Developments in World Seismology, vol. 2, pp. 19-23, 2007 (Chinese).

[8] K. Wang, Influence of blasting excavation on nearby corroded pipeline and risk assessment in coastal areas [Ph.D. thesis], Dalian University of Technology, Dalian, China, 2013, (Chinese).

[9] H.-Y. Liu, X.-W. Niu, A.-G. Ruan, Z.-L. Wu, J.-B. Li, and S. Pan, "Analysis of seismic signals of ocean bottom seismometer," Joural of Tropical Oceangraphy, vol. 31, no. 3, pp. 90-96, 2012 (Chinese).

[10] R. S. Jacobson, L. M. Dorman, and G. M. Purdy, "Ocean bottom seismometer facilities avilable," Journal of Physics of the Earth, vol. 43, pp. 599-618, 1995.

[11] D. G. Levchenko, "Effect of the water-saturated sediment layer on recording seismic signals with a bottom seismometer," Oceanology, vol. 46, no. 5, pp. 739-750, 2006.

[12] A. Trehu, "Coupling of ocean bottom seismometers to sediment: results of tests with U.S. geological survey ocean bottom seismometers," Bulletin of the Seismological Society of America, vol. 75, no. 1, pp. 271-289, 1985.

[13] F.-Y. Xue and S.-K. Zhai, "Ocean drilling in the 21st centuryIODP," Marine Geology \& Quaternary Geology, vol. 22, no. 1, pp. 109-114, 2002 (Chinese).

[14] E. Araki, M. Shinohara, S. Sacks et al., "Improvement of seismic observation in the ocean by use of seafloor boreholes," Bulletin of the Seismological Society of America, vol. 94, no. 2, pp. 678690, 2004.

[15] H.-T. Li, L. Zhao, Y.-C. Ning et al., "Testing and analyzing pressure produced by submarine explosion with spherical charges," Engineering Blasting, vol. 11, no. 1, pp. 16-21, 2005 (Chinese).

[16] D.-Q. Zhu, X.-L. Li, X.-J. Zhan et al., "Observation and analysis of blasting vibration on the right banks high slope of longtan hydropower," Blasting, vol. 19, no. 4, pp. 65-67, 2002 (Chinese).

[17] R. E. Goodman, Methods of Geological Engineering in Discontinuous Rocks, West Publishing, St. Paul, Minn, USA, 1976. 

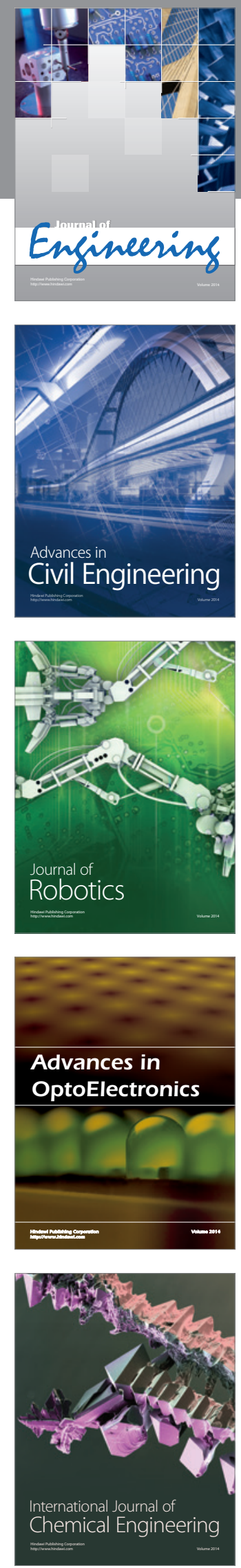

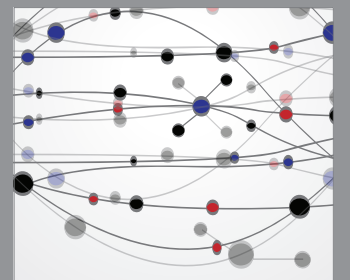

The Scientific World Journal
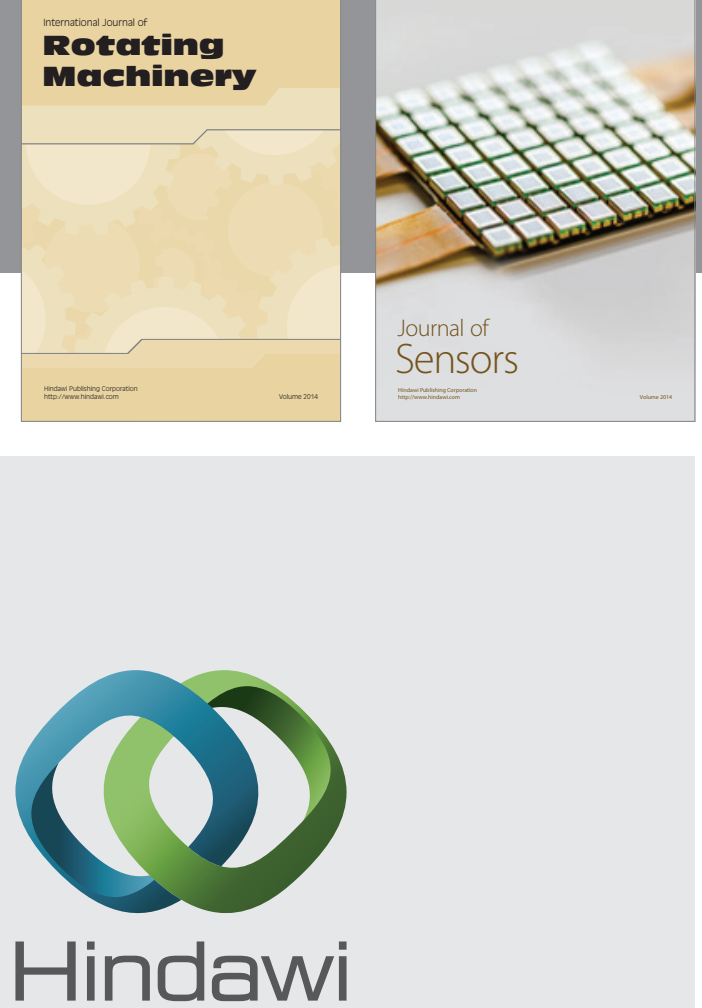

Submit your manuscripts at http://www.hindawi.com
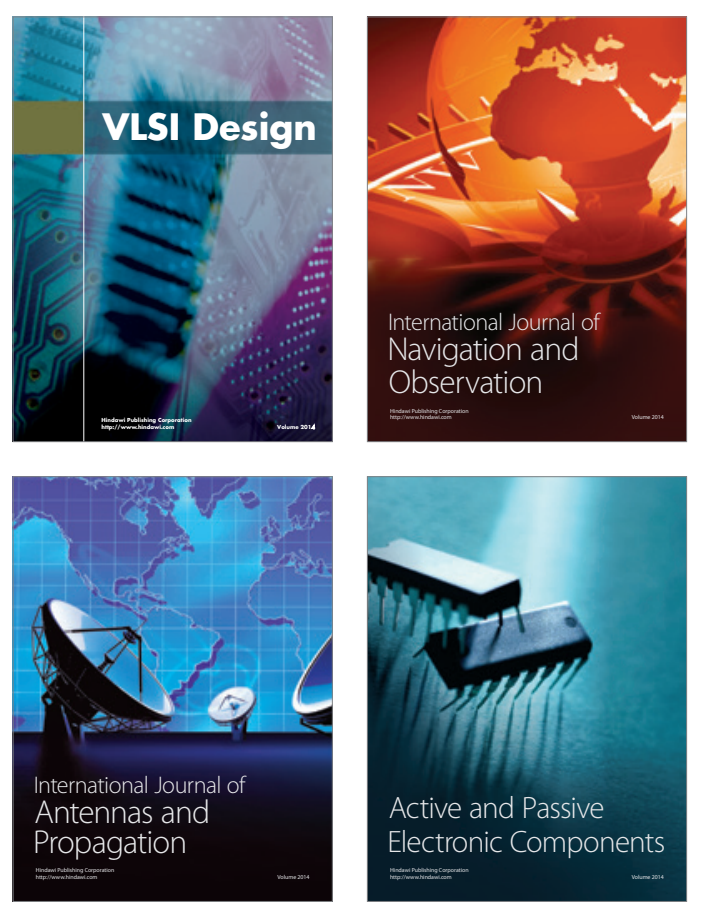
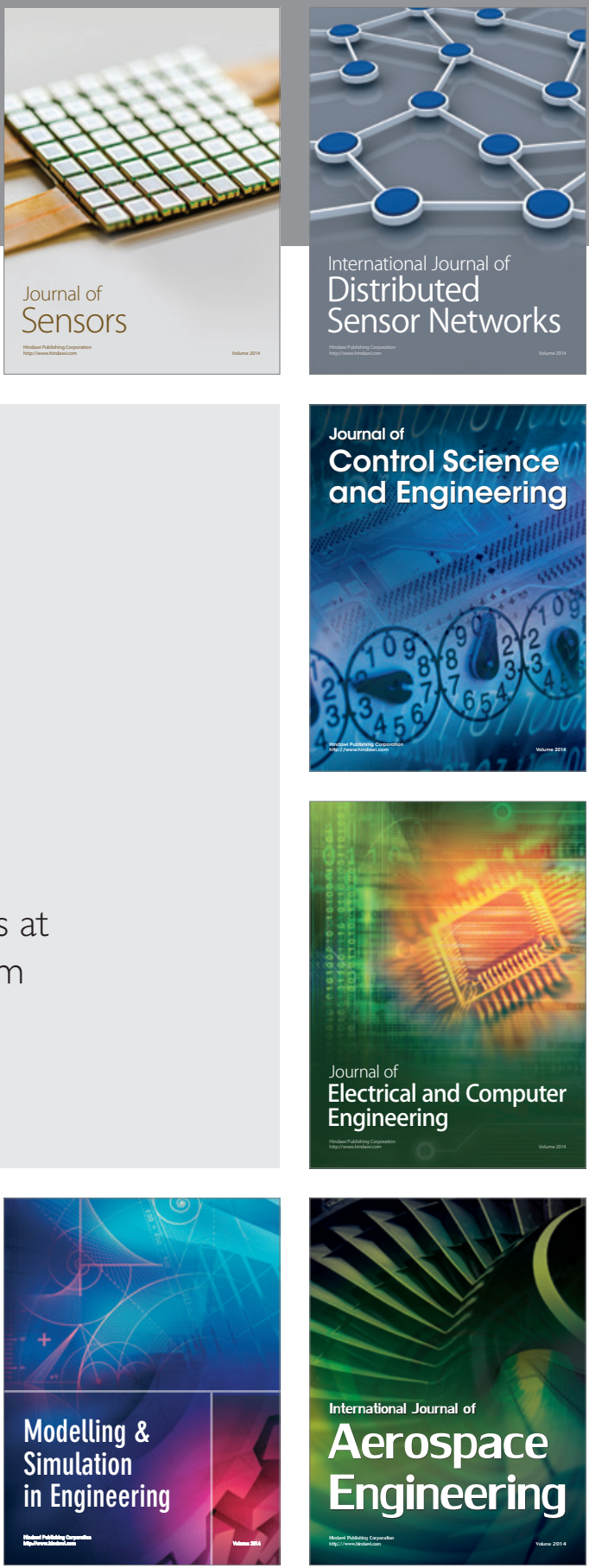

Journal of

Control Science

and Engineering
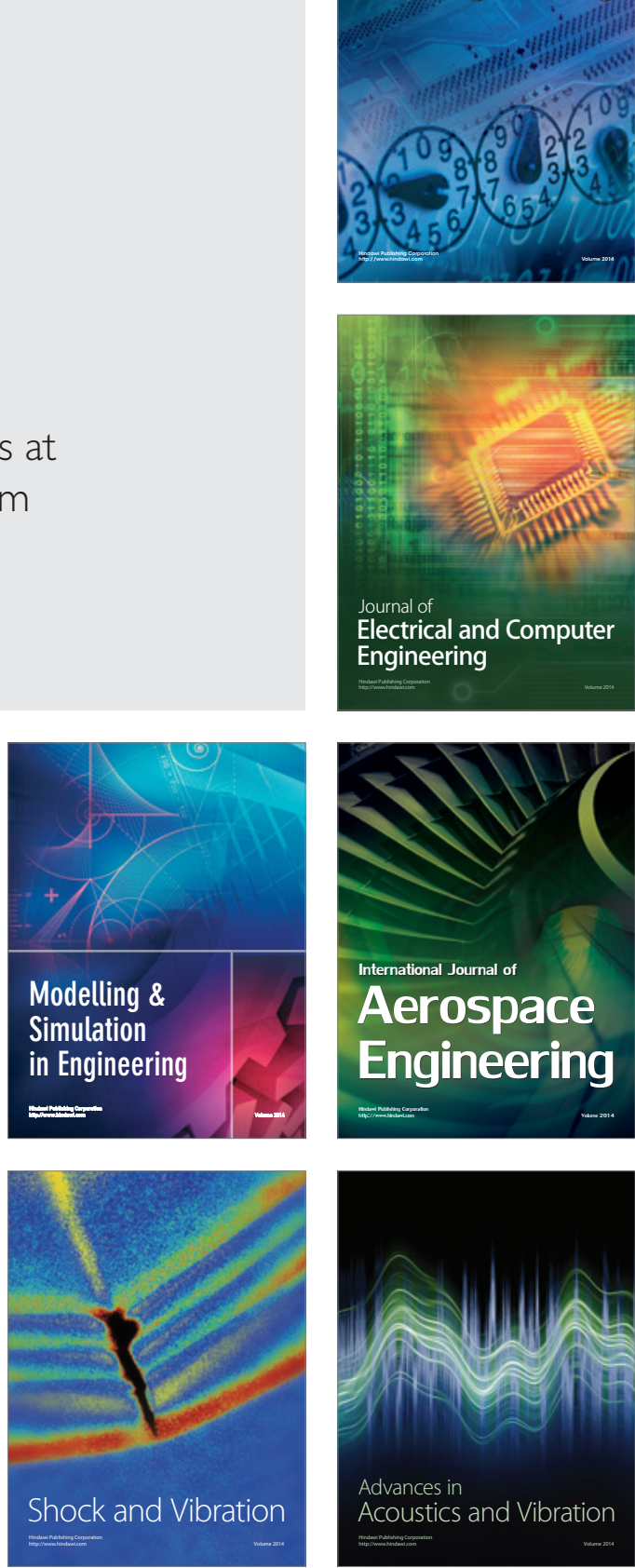